\title{
Counteracting Electrode Shifts in Upper-Limb Prosthesis Control via Transfer Learning
}

\author{
Cosima Prahm*1,2, Alexander Schulz*3, Benjamin Paaßen*3, Johannes Schoisswohl ${ }^{1,2}$, Eugenijus \\ Kaniusas $^{2}$, Georg Dorffner ${ }^{4}$, Barbara Hammer ${ }^{3}$, and Oskar Aszmann ${ }^{1}$ \\ ${ }^{1}$ Christian Doppler Laboratory for Restoration of Extremity Function, Department of Surgery, at \\ the Medical University of Vienna, Austria \\ ${ }^{2}$ Institute of Electrodynamics, Microwave and Circuit Engineering, Vienna University of \\ Technology, Austria \\ ${ }^{3}$ Center of Excellence Cognitive Interaction Technology, Bielefeld, Germany \\ ${ }^{4}$ Center for Medical Statistics, Informatics and Intelligent Systems at the Medical University of \\ Vienna, Austria
}

Preprint of the paper Prahm, Schulz, Schoisswohl, et al. (2019) as provided by the authors. The original can be found at doi:10.1109/TNSRE.2019.2907200

Authors marked with * contributed equally.

\section{Abstract}

Research on machine learning approaches for upper limb prosthesis control has shown impressive progress. However, translating these results from the lab to patient's everyday lives remains a challenge, because advanced control schemes tend to break down under everyday disturbances, such as electrode shifts. Recently, it has been suggested to apply adaptive transfer learning to counteract electrode shifts using as little newly recorded training data as possible.

In this paper, we present a novel, simple version of transfer learning and provide the first user study demonstrating the effectiveness of transfer learning to counteract electrode shifts. For this purpose, we introduce the novel Box and Beans test to evaluate prosthesis proficiency and compare user performance with an initial simple pattern recognition system, the system under electrode shifts, and the system after transfer learning. Our results show that transfer learning could significantly alleviate the impact of electrode shifts on user performance in the Box and Beans test.

\section{Introduction}

In recent years, machine learning control for upper limb prostheses has made significant progress, driven by new control algorithms (Janne M. Hahne, Biebmann, et al. 2014 Ning Jiang et al. 2014: Muceli, I. Vujaklija, et al. 2017; Prahm, Schulz, Paaßen, et al. 2017; Aidan D. Roche et al. 2014), new training paradigms, such as co-adaptive training, virtual reality, and games (J. M. Hahne et al. 2015, Prahm, Ivan Vujaklija, et al. 2017; Aidan D. Roche et al. 2014), new surgical techniques, such as targeted muscle reinnervation (Todd et al. 2009; Aidan D. Roche et al. 2014), new prosthetic devices (Belter et al. 2013 Controzzi et al. 2017), and new electrodes to record user's control signal, such as highdensity electrode grids (Daley et al. 2012 Muceli, N. Jiang, and D. Farina 2014) or implantable sensors (Janne M. Hahne, Dario Farina, et al. 2016, OrtizCatalan et al. 2012 Pasquina et al. 2015). However, translating many promising results from the lab to an amputee's everyday life remains a challenge due to various sources of disturbance, such as posture changes, sweating, weight of grasped objects, long term changes, or electrode shifts (D. Farina et al. 2014 L. Hargrove, Englehart, and Hudgins 2008, Khushaba et al. 2014, Young, L. J. Hargrove, and 




Figure 1: An illustration of an 8 electrode grid before (transparent) and after (opaque) an electrode shift. The arrows indicate the direction of electrode shift and the virtual correction, respectively. The cross section of the arm is adapted from the 1921 German edition of "Anatomie des Menschen", which is in the public domain.

Kuiken 2011; Hwang, Janne Mathias Hahne, and Müller 2017). Indeed, the negative effects of such disturbances on prosthesis control may be so severe that users become less confident when using the prosthesis, or abandon their prosthesis altogether (Biddiss and Chau 2007; D. Farina et al. 2014). Therefore, counteracting disturbances such as electrode shifts is a key challenge in contemporary prosthesis research (D. Farina et al. 2014). In this work, we focus particularly on transversal electrode shifts (see Figure 1) which have been identified as more impactful compared to longitudinal shifts (Young, L. J. Hargrove, and Kuiken 2012).

Multiple approaches in the past have tried to cope with electrode shifts. For example, Hargrove, Englehart, and Hudghins have suggested to record training data in all plausible shift conditions to achieve a pattern recognition model that is invariant against shifts (L. Hargrove, Englehart, and Hudgins 2008). However, this approach is limited to disturbances that are present in the training data, and to disturbances which do not introduce class overlap.

Alternatively, past work has recommended to use different features of the myoelectric signal which are more robust than time-domain features with respect to electrode shifts. In particular, Hargrove et al. as well as Young et al. propose auto-regressive features (L. Hargrove, Englehart, and Hudgins 2008, Young, L. J. Hargrove, and Kuiken 2012) and Khushaba et al. recommend spectral features (Khushaba et al. 2014). While both approaches improve classifi- cation accuracy, they are still severely affected by shifts. Therefore, we believe that other approaches are needed in addition to these feature-based techniques.

Further, several authors have suggested alternative sensors to increase the robustness against electrode shifts. For instance, Muceli et al. as well as Pan et al. propose high-density electrode grids and subsequently extracted features (Muceli, N. Jiang, and D. Farina 2014. L. Pan et al. 2015). An invasive option is offered by implantable sensors which are not subject to electrode shifts because they directly surface the muscle (Janne M. Hahne, Dario Farina, et al. 2016; Ortiz-Catalan et al. 2012; Pasquina et al. 2015). However, to date, neither high-density electrodes, nor implantable sensors have been very common in commercial or research systems for prosthesis control(D. Farina et al. 2014).

Finally, there are approaches which adapt the machine learning model to the disturbed data. In particular, Amsüss et al. propose a post-processing approach which rejects uncertain decisions by the machine learning model, yielding smoother and more accurate decisions (S. Amsüss et al. 2014). Vidovic et al. interpolate between the means and covariances of the original training data and a small set of disturbed training data to adapt their machine learning model to the disturbed condition (Vidovic et al. 2015). Finally, Prahm et al. as well as Paassen et al. propose to learn a transformation which cleans up the disturbed data, such that the original model is applicable again (Prahm, Paaßen, et al. 2016 Paaßen, Schulz, J. Hahne, et al. 2018). The key benefit of these latter approaches is that they only need to model the effect of the disturbance while leaving the controller itself as is. In this paper, we build upon this transfer learning approach and simplify it for easier application in practice.

More specifically, we contribute a new adaptation scheme for transversal electrode shifts in regular electrode grids, which is compatible with any machine learning model based on a loss function. Secondly, we provide a novel, easily accessible, and cost effective way to evaluate prosthetic proficiency in form of the virtual Box and Beans test, which is based on the popular Box and Blocks test. Finally, we present the first user study on transfer learning for counteracting transversal electrode shifts. Our results show that transfer learning could significantly 
improve user performance in the Box and Beans test.

\section{Method}

In our study, we evaluated a prosthetic pattern recognition user interface in three conditions, namely an initial, undisturbed condition (A), a condition disturbed by electrode shift (B), and a condition where transfer learning is applied after electrode shift (C). Note that we did not directly compare to a reference method from the literature because these methods are either complementary to our method (L. Hargrove, Englehart, and Hudgins 2008, Young, L. J. Hargrove, and Kuiken 2012, Khushaba et al. 2014; S. Amsüss et al. 2014), apply to different sensor settings (Muceli, N. Jiang, and D. Farina 2014; L. Pan et al. 2015 Janne M. Hahne, Dario Farina, et al. 2016; Ortiz-Catalan et al. 2012 Pasquina et al. 2015), or need calibration data from all movements whereas our approach requires only a calibration set for a subset of movements (Vidovic et al. 2015).

We first introduce our signal extraction (Section 2.1.1) and pattern recognition (Section 2.1.2) schemes and then describe our proposed transfer learning approach for condition C (Section 2.1.3). In all conditions, we evaluated prosthetic proficiency by a novel Box and Beans test (Section 2.2.1) and we evaluated the users' attitudes toward the system by a questionnaire (Section 2.2.2). Finally, we present the study design in more detail (Section 2.2.3).

\subsection{Algorithm description}

\subsubsection{Signal extraction}

We recorded the myoelectric signal via the 8-channel Thalmic Myo armband with a sampling rate of $200 \mathrm{~Hz}$. The 8 channels are ordered in a regular grid around the forearm, as illustrated in Figure 1. Due to its comparably low sampling rate, pattern recognition is harder in the Thalmic Myo compared to professional systems (Phinyomark, N. Khushaba, and Scheme 2018), such that satisfactory results on the Thalmic Myo are likely to translate well to higherquality setups.

Following Hahne et al. (Janne M. Hahne, Biebmann, et al. 2014), we pre-processed the data in each channel by a $50 \mathrm{~Hz}$ comb filter to avoid potential power line interference via capacitive coupling, and by computing the logarithm of the variance on windows of $120 \mathrm{~ms}$ with $40 \mathrm{~ms}$ overlap. According to Hahne et al., the log variance is roughly linearly related to the strength of the motion intent, thus promising a good representation for motion classification. In the following, we denote the 8 dimensional vector of log-variances per channel at time step $t$ as $\mathrm{x}_{\mathrm{t}} \in \mathcal{X} \subset \mathbb{R}^{8}$.

\subsubsection{Pattern Recognition}

Our system should be able to infer the intended motion $\mathbf{y}_{\mathrm{t}}$ from the current signal $\mathbf{x}_{\mathrm{t}}$. In our case, we are interested in motions in two degrees of freedom $(\mathrm{DoF}) \mathbf{y}_{\mathrm{t}}=\left(\mathrm{y}_{\mathrm{t}}^{1}, \mathrm{y}_{\mathrm{t}}^{2}\right)$, namely wrist rotation encoded by $y_{t}^{1}$ and hand opening/closing by $y_{t}^{2}$. Thereby $y_{t}^{1}, y_{t}^{2} \in\{-1,0,1\}$, where a value of 0 implies no movement in that DoF, $\mathbf{y}_{\mathrm{t}}=(-1,-1)$ encodes wrist pronation combined with hand closing, and $\mathbf{y}_{\mathrm{t}}=(1,1)$ encodes wrist supination combined with hand opening.

To solve this task we utilize Generalized Matrix Learning Vector Quantization (GMLVQ) (Schneider, Biehl, and Hammer 2009). In particular, we train two GMLVQ models, one per DoF r, which are executed in parallel to achieve an independent classification in both DoFs. GMLVQ works by initializing $\mathrm{m}$ (hyperparameter) so-called prototypes $\mathbf{w}_{1}^{\mathrm{r}}, \ldots, \mathbf{w}_{\mathrm{m}}^{\mathrm{r}} \in \mathcal{X}$ in the space of the data $\mathcal{X}$, each of which is associated with one corresponding movement $\mathrm{y}_{\mathrm{t}}^{\mathrm{r}} \in\{-1,0,1\}$. The term prototype refers to the fact that every EMG pattern $\mathbf{x}_{\mathrm{t}}$ in the data set should be closest to a prototype corresponding to the actual movement $\mathrm{y}_{\mathrm{t}}^{\mathrm{r}}$ and further away from prototypes for different movements. We achieve this property by minimizing the following loss function:

$$
\sum_{\mathrm{t}} \ell\left(\mathbf{x}_{\mathrm{t}}, \mathrm{y}_{\mathrm{t}}^{\mathrm{r}}\right) \quad \text { where } \ell\left(\mathbf{x}_{\mathrm{t}}, \mathrm{y}_{\mathrm{t}}^{\mathrm{r}}\right)=\frac{d^{+}\left(\mathbf{x}_{\mathrm{t}}\right)-d^{-}\left(\mathbf{x}_{\mathrm{t}}\right)}{d^{+}\left(\mathrm{x}_{\mathrm{t}}\right)+d^{-}\left(\mathbf{x}_{\mathrm{t}}\right)}
$$

where $d^{+/-}\left(\mathbf{x}_{\mathrm{t}}\right)$ is the distance from $\mathbf{x}_{\mathrm{t}}$ to the nearest prototype with the same/different label, respectively. The distance between a data point $\mathbf{x}_{\mathrm{t}}$ and a prototype $\mathbf{w}_{\mathrm{k}}^{\mathrm{r}}$ with $\mathrm{k} \in\{1, \ldots, \mathrm{m}\}$ and $\mathrm{r} \in\{1,2\}$ is quantified as follows.

$$
d\left(\mathbf{x}_{\mathrm{t}}, \mathbf{w}_{\mathrm{k}}^{\mathrm{r}}\right)=\left(\mathbf{x}_{\mathrm{t}}-\mathbf{w}_{\mathrm{k}}^{\mathrm{r}}\right)^{\top}\left(\boldsymbol{\Omega}^{\mathrm{r}}\right)^{\top} \boldsymbol{\Omega}^{\mathrm{r}}\left(\mathbf{x}_{\mathrm{t}}-\mathbf{w}_{\mathrm{k}}^{\mathrm{r}}\right),
$$

where the matrix $\Omega^{\mathrm{r}} \in \mathbb{R}^{8 \times 8}$ is a linear projection learned by the model to facilitate classification. To optimize the loss (1), we utilize the limited-memory Broyden-Fletcher-Goldfarb-Shanno 


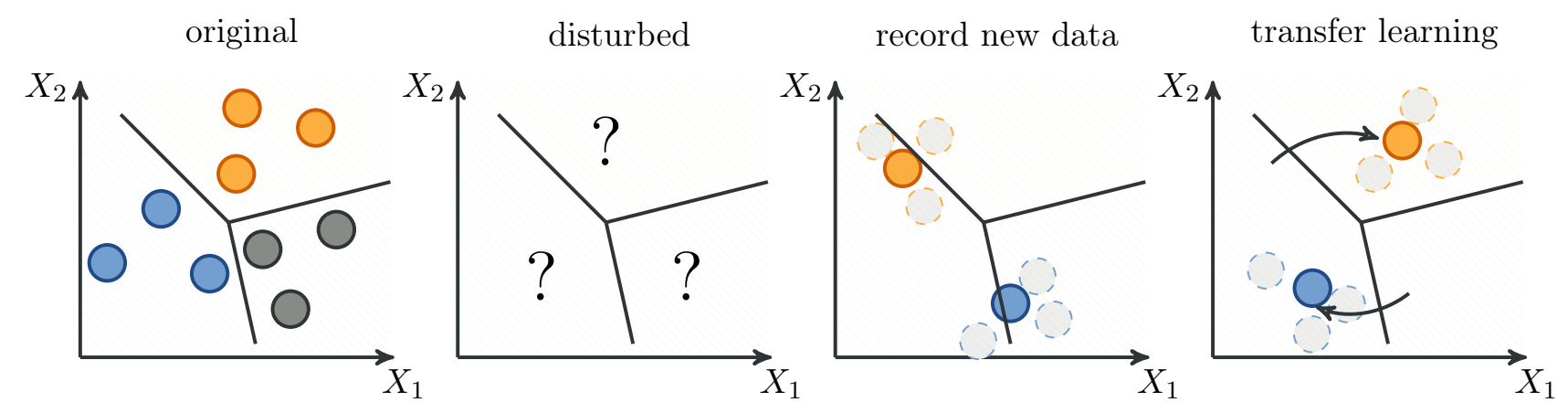

Figure 2: Overview over the considered transfer learning setting, from left to right. We first train a pattern recognition model on data recorded under lab conditions, i.e. from the source domain (original). The colors of the points indicate their according class. Then, the incoming data is disturbed via an electrode shift such that the model may not be appropriate (disturbed). To estimate the disturbance, we record a few new data instances from a few of the classes in the disturbed condition, i.e. in the target domain (record new data). Grey points indicate possible positions of future data. Finally, the transfer learning step utilizes the gathered data to learn a transformation of disturbed data, such that the original classification model is applicable again (transfer learning).

(BFGS) algorithm (Fletcher 1987) and the GMLVQ implementation provided as part of the SOM toolbox 1

After we obtain a GMLVQ model for a data set, we can predict the label for a new instance $\mathbf{x}_{\mathrm{t}}$ by computing the distances $d\left(\mathbf{x}_{\mathrm{t}}, \mathbf{w}_{\mathrm{k}}^{\mathrm{r}}\right)$ to all prototypes $\mathbf{w}_{1}^{\mathrm{r}}, \ldots, \mathbf{w}_{\mathrm{m}}^{\mathrm{r}}$ and selecting the label of the closest prototype.

Benefits of GMLVQ include good generalization properties due to low model complexity, interpretable components (the prototypes, the relevance assigned to each feature by $\boldsymbol{\Omega}^{\mathrm{r}}$ ), and a low computational complexity (Schneider, Biehl, and Hammer 2009). As such, the GMLVQ algorithm provides a model which is feasible for rapid classification in systems with very limited computational resources, such as embedded systems in a prosthetic device.

Now that we have obtained a GMLVQ model, our main challenge is to adapt the model to electrode shifts.

\subsubsection{Transfer Learning}

Our aim is to counteract electrode shifts via transfer learning. In general, transfer learning is concerned with transferring knowledge from some source domain or task to a different target domain or task (S. J. Pan and Yang 2010). In our case, we have

\footnotetext{
${ }_{1}^{1}$ https://github.com/ilarinieminen/SOM-Toolbox
}

already learned a model $f: \mathcal{X} \rightarrow\{-1,0,1\}^{2}$ which infers intended motions from EMG data. However, in an everyday situation, electrode displacements change the recording of user motions such that these are no longer mapped to the same positions in $\mathcal{X}$, but to different ones which we refer to as the disturbed data space $\hat{\mathcal{X}} \subset \mathbb{R}^{8}$. Here, our model $f$ makes incorrect predictions, i.e. $f\left(\hat{\mathbf{x}}_{\mathrm{t}}\right) \neq \mathbf{y}_{\mathrm{t}}$, with $\hat{\mathbf{x}}_{\mathrm{t}} \in \hat{\mathcal{X}}$.

In our work, we follow the basic approach of Paaßen et al., who propose to exploit the relationship between the source data and target data in order to apply the source model $f$ to the target data. If this relationship is simple compared to the model $f$ itself, learning the relationship should also be simpler compared to learning a new model for the disturbed condition (Paaßen, Schulz, and Hammer 2016 Paaßen, Schulz, J. Hahne, et al. 2018). Figure 2 illustrates this transfer learning scenario.

While previous work has applied an ad-hoc linearity assumption (Paaßen, Schulz, J. Hahne, et al. 2018. Prahm, Paaßen, et al. 2016), we explore the relationship between the undisturbed and disturbed data in more detail and exploit additional knowledge about the domain. In particular, we know that the data is recorded via a regular 8 electrode grid, where the distance between the electrodes is held roughly equal by the armband (see Figure 1). In formal terms, we assume that the jth electrode sits at position $\mathrm{j} \cdot 45^{\circ}$ around the forearm, where 


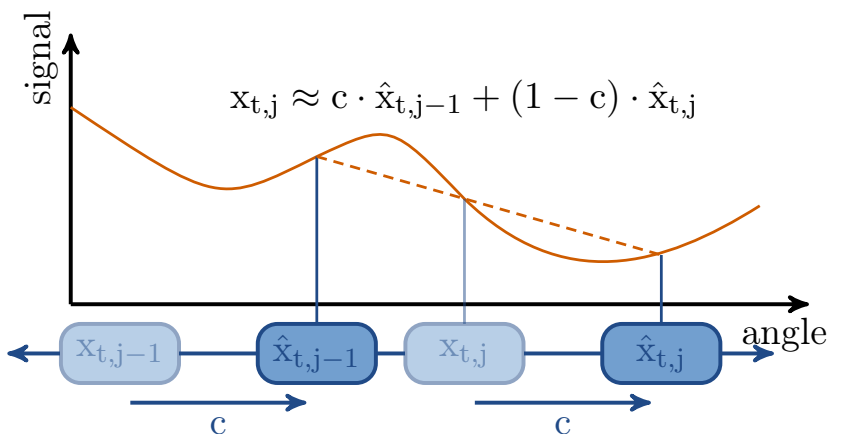

Figure 3: An illustration of our proposed scheme to estimate the undisturbed features of the jth electrode $\mathrm{x}_{\mathrm{t}, \mathrm{j}}$ from the disturbed features $\hat{\mathrm{x}}_{\mathrm{t}, \mathrm{j}-1}$ and $\hat{\mathrm{x}}_{\mathrm{t}, \mathrm{j}}$ after an electrode shift by $\mathrm{c} \in[0,1]$ electrodes. The $\mathrm{x}-$ axis displays the angle around the forearm, the $\mathrm{y}$ axis the feature amplitude. Note that we assume that the feature can be linearly interpolated between the electrodes.

$\mathrm{j} \in\{1, \ldots, 8\}$. Now, let $\phi(\mathrm{t}, \theta)$ denote the features corresponding to time step $\mathrm{t}$ and angle $\theta$ around the forearm, let $x_{t, j}$ denote the features of electrode $j$ at time step $t$, and let $\hat{x}_{t, j}$ denote the features of electrode $\mathrm{j}$ at time step $\mathrm{t}$ after an electrode shift. For all $\mathrm{t}$ and all $\mathrm{j} \in\{1, \ldots, 8\}$ it holds per definition $\mathrm{x}_{\mathrm{t}, \mathrm{j}}=\phi\left(\mathrm{t}, \mathrm{j} \cdot 45^{\circ}\right)$. Further, for any electrode shift by $\mathrm{c} \in[0,1]$ electrodes we obtain $\hat{\mathrm{x}}_{\mathrm{t}, \mathrm{j}}=\phi\left(\mathrm{t},[\mathrm{j}+\mathrm{c}] \cdot 45^{\circ}\right)$ for all $\mathrm{j} \in\{1, \ldots, 8\}$.

Based on this setup, and the assumption that features change linearly between neighboring electrodes, we can estimate the features at the jth unshifted electrode $x_{t, j}$ based on the features at the $j$ th shifted electrode $\hat{x}_{t, j}$ and the features at the $j-1$ th shifted electrode $\hat{\mathrm{x}}_{\mathrm{t}, \mathrm{j}-1}$ as follows (see Figure 3 ).

$$
\begin{aligned}
& \mathrm{x}_{\mathrm{t}, \mathrm{j}}=\phi\left(\mathrm{t}, \mathrm{j} \cdot 45^{\circ}\right) \\
= & \phi\left(\mathrm{t},[\mathrm{j}+\mathrm{c}] \cdot 45^{\circ}\right)-\left(\frac{\partial}{\partial \mathrm{c}} \phi\left(\mathrm{t},[\mathrm{j}+\mathrm{c}] \cdot 45^{\circ}\right)\right) \cdot \mathrm{c} \cdot 45^{\circ} \\
= & \hat{\mathrm{x}}_{\mathrm{t}, \mathrm{j}}-\frac{\phi\left(\mathrm{t},[\mathrm{j}+\mathrm{c}] \cdot 45^{\circ}\right)-\phi\left(\mathrm{t},[\mathrm{j}-1+\mathrm{c}] \cdot 45^{\circ}\right)}{[\mathrm{j}+\mathrm{c}] \cdot 45^{\circ}-[\mathrm{j}-1+\mathrm{c}] \cdot 45^{\circ}} \cdot \mathrm{c} \cdot 45^{\circ} \\
= & \hat{\mathrm{x}}_{\mathrm{t}, \mathrm{j}}-\left(\hat{\mathrm{x}}_{\mathrm{t}, \mathrm{j}}-\hat{\mathrm{x}}_{\mathrm{t}, \mathrm{j}-1}\right) \cdot \mathrm{c} \\
= & (1-\mathrm{c}) \cdot \hat{\mathrm{x}}_{\mathrm{t}, \mathrm{j}}+\mathrm{c} \cdot \hat{\mathrm{x}}_{\mathrm{t}, \mathrm{j}-1}
\end{aligned}
$$

Note that the equality in the second and third line rely on our linearity assumption. In other words, we estimate $\mathrm{x}_{\mathrm{t}, \mathrm{j}}=\phi\left(\mathrm{t}, \mathrm{j} \cdot 45^{\circ}\right)$ via a first-order Taylor expansion of $\phi$ at point $\hat{\mathrm{x}}_{\mathrm{t}, \mathrm{j}}=\phi\left(\mathrm{t},[\mathrm{j}+\mathrm{c}] \cdot 45^{\circ}\right)$ and a finite-difference approximation of the derivative using point $\hat{\mathrm{x}}_{\mathrm{t}, \mathrm{j}-1}$.
If we generalize this result over all electrodes, we obtain $\mathbf{x}_{\mathrm{t}}=\mathbf{T}(\mathrm{c}) \cdot \hat{\mathbf{x}}_{\mathrm{t}}$, where $\mathbf{T}(\mathrm{c})$ is the matrix with entries $\mathbf{T}(\mathrm{c})_{\mathrm{i}, \mathrm{i}}=1-\mathrm{c}, \mathbf{T}(\mathrm{c})_{\mathrm{i}+1, \mathrm{i}}=\mathrm{c}, \mathbf{T}(\mathrm{c})_{1,8}=\mathrm{c}$ and $\mathbf{T}(\mathrm{c})_{\mathrm{i}, \mathrm{j}}=0$ otherwise. We can repeat this argument for the case of electrode shifts in the inverse direction, which we denote by a negative $c$, in which case we obtain the equation $\mathbf{x}_{\mathrm{t}}=\mathbf{T}(-\mathrm{c})^{\top} \cdot \hat{\mathbf{x}}_{\mathrm{t}}$.

So far, we considered electrode shifts of up to one electrode. Larger shifts can be analysed analogously, i.e. reconstructing the signal of an old electrode by the signals of its two new neighbors. For general $\mathrm{c} \in \mathbb{R}$ we obtain the matrix $\mathbf{T}(\mathrm{c})$ as $\mathbf{T}(\mathrm{c}-8)$ if $\mathrm{c} \geq 8$, as $\mathbf{T}(-c)^{\top}$ if $\mathrm{c}<0$, and otherwise:

$$
\mathbf{T}(\mathrm{c})_{\mathrm{i}, \mathrm{j}}= \begin{cases}1-\bmod (\mathrm{c}, 1) & \text { if } \mathrm{j}=\mathrm{i}-\lfloor\mathrm{c}\rfloor \\ 1-\bmod (\mathrm{c}, 1) & \text { if } \mathrm{j}=\mathrm{i}+8-\lfloor\mathrm{c}\rfloor \\ \bmod (\mathrm{c}, 1) & \text { if } \mathrm{j}=\mathrm{i}-\lceil\mathrm{c}\rceil \\ \bmod (\mathrm{c}, 1) & \text { if } \mathrm{j}=\mathrm{i}+8-\lceil\mathrm{c}\rceil \\ 0 & \text { otherwise }\end{cases}
$$

where $\bmod (\mathrm{c}, 1)$ is $\bmod (\mathrm{c}-1,1)$ if $\mathrm{c} \geq 1$ and $\mathrm{c}$ if $\mathrm{c}<1,\lfloor\mathrm{c}\rfloor$ is the largest integer which is at least as small as $\mathrm{c}$ and $\lceil\mathrm{c}\rceil$ is the smallest integer larger than c.

The only remaining challenge now is to estimate the single parameter $\mathrm{c}$ from data. More precisely, we wish to identify a parameter $\mathrm{c}$ such that our existing model $f$ performs as well as possible on the disturbed data after applying $\mathbf{T}(\mathrm{c})$. Hence, we can optimize c by solving the following minimization problem.

$$
\arg \min _{\mathrm{c}} \frac{1}{2} \sum_{\mathrm{r}=1}^{2} \sum_{\mathrm{t}} \ell\left(\mathbf{T}(\mathrm{c}) \cdot \hat{\mathbf{x}}_{\mathrm{t}}, \mathrm{y}_{\mathrm{t}}^{\mathrm{r}}\right)
$$

where $\ell$ may be any loss function which quantifies the deviation between the prediction of a model for the input $\mathbf{T}(\mathrm{c}) \cdot \hat{\mathbf{x}}_{\mathrm{t}}$ and the desired motion $\mathbf{y}_{\mathrm{t}}$, in our case the GMLVQ loss from Equation (1). To solve this problem we can simply sample $\mathrm{c}$ from a

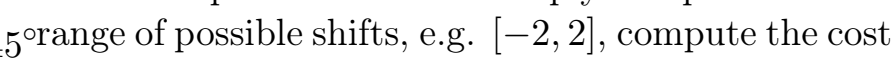
function (3) for each and select the c with minimum cost.

An example of the selection procedure is depicted in Figure 4. The $\mathrm{x}$-axis depicts different $\mathrm{c}$ values, where a positive sign implies corrections for lateral shifts and a negative sign for medial shifts of amount $|c|$. The $y$-axis denotes the value of the cost function (3) that we wish to optimize. The orange and blue curve mark the according values for the two degrees of freedom in our system, i.e. hand open/close 


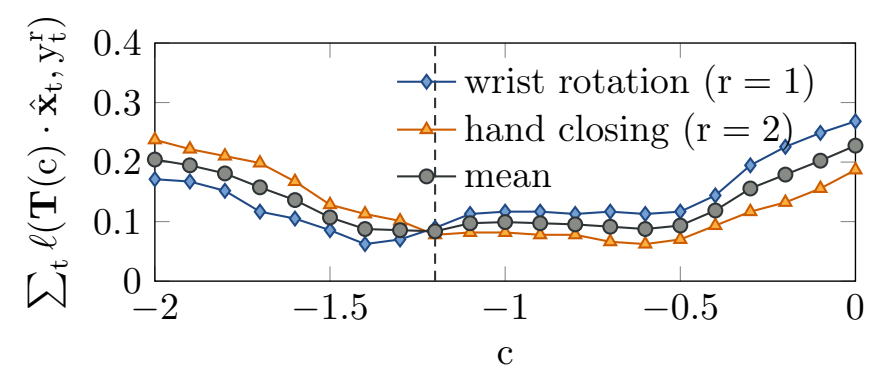

Figure 4: An example for the selection procedure for the best value of $\mathrm{c}$. The $\mathrm{X}$-axis denotes different values of $\mathrm{c}$ where a positive sign indicates a lateral, and a negative sign indicates a medial shift. The y-Axis displays the value of the cost function (3) for both degrees of freedom (wrist rotation in blue and hand opening/closing in orange), as well as the mean of both (gray). The gray, dashed, vertical line indicates the $\mathrm{c}$ with minimum error.
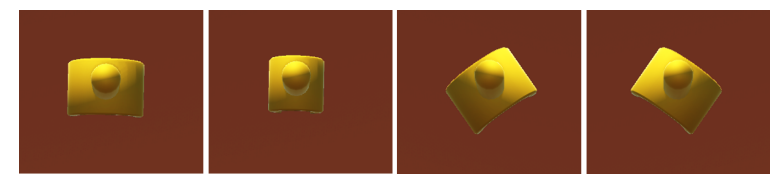

Figure 5: The different grasp-forms the virtual grasper can perform: open, close, rotate left, rotate right and the simultaneous combinations of those.

and supination/pronation, the black curve is their average. Finally, the vertical dashed black line indicates the selected best value of c, according to the minimum of the black curve. If there are multiple minimum values, we select $\mathrm{c}$ as the median of those.

Note that the estimated c does not need to exactly correspond to the ground truth $(\mathrm{c}=-0.8$, in this case). Yet, we can still achieve a low predictive error in a range around the ground truth $\mathrm{c}$ value $(\mathrm{c} \in$ $[-1.4,-0.5]$, in this case).

\subsection{Experiment description}

\subsubsection{From Blocks to Beans - the Box and Beans Test}

The Box and Blocks test is a quick and common tool in physiotherapy to measure unilateral manual dexterity. Patients are scored based on the number of blocks they are able to transfer within 60 s from one compartment to the opposite one. The two compartments are separated from another by a board which is higher than the box' walls (Math- iowetz et al. 1985). A common way to test prosthetic proficiency with a novel algorithm is to fit the patient with a personalized socket and to provide able-bodied participants with a hybrid prosthesis attached to their healthy arm (Aidan Dominic Roche et al. 2015). As both solutions are impractical and cost-intensive, we propose to implement the Box and Blocks test in a virtual environment instead 2 Because a direct 2D implementation of the Box and Blocks test would mean losing one DoF (either rotation for transradial prosthesis users or elbow function for transhumeral users), we replaced the blocks by beans. With this bean or barbell shape, the users of the virtual Box and Beans test were coerced to rotate their virtual grasper in order to catch the beans because the grasper needs to slide over the bulky endings of the bean before attaching to the thin middle part. Using the EMG signals, the grasper could be opened, closed, rotated left and right or perform any expedient simultaneous combination of these movements (see Figure 5p. The grasper was controlled by pattern recognition as described above, where each movement was executed at a constant speed. The built-in gyroscope of the Myo armband allowed the users to freely move their arm, just like in the real Box and Blocks test, and thereby maneuver the grasper across the screen. When only one bean was left in the start compartment, three new, randomly oriented, colored and positioned beans would spawn in the start compartment so that there would never be a shortage of beans (see Figure 6).

\subsubsection{Questionnaire}

A modified version of the intrinsic motivation inventory (IMI) questionnaire was given to the participants after finishing performing the Box and Beans test in each condition in order to assess the experience of the game control and whether participants noticed differences in the control method at all (Ryan 1982). The IMI included in this study consisted of 19 questions corresponding to four subscales: Enjoyment, perceived pressure, effort and usefulness. The questions were adapted to fit the study by exchanging "working" and "doing" from the original IMI with "using this control". Participants could mark their answer on a 7-point Likert scale ranging from

\footnotetext{
${ }^{2}$ Refer to https://github.com/joeschman/BoxAndBeans for the source code
} 


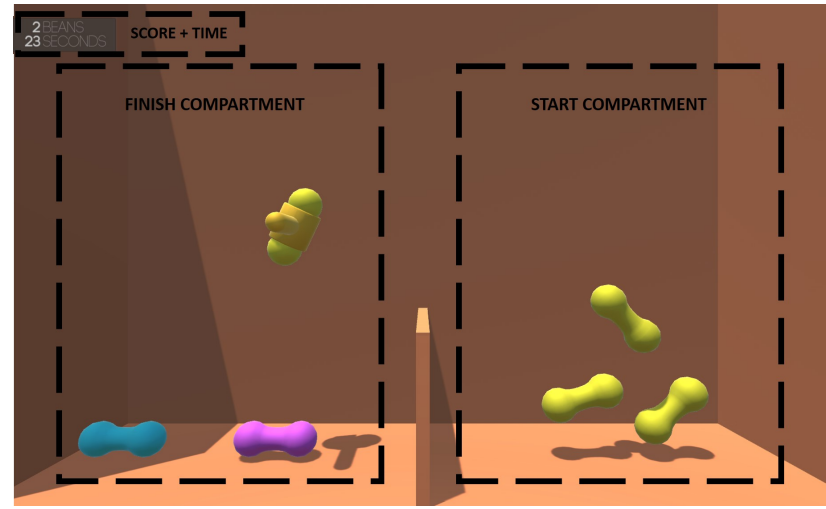

Figure 6: Layout of the Box and Beans test: The beans are to be transferred from the start compartment to the finish compartment. They have to be passed over the wall between both compartments and fall to or be placed on the the ground to successfully score a point. The score and remaining time can be seen at the top left of the screen.

1 ("strongly disagree") to 7 ("strongly agree").

\subsubsection{Study design}

Four upper limb amputees on a transradial level (below elbow) and nine able-bodied participants took part in this study. They were each seated in front of a computer screen showing the Box and Beans test and wearing the Myo Armband by Thalmic Labs around their forearm (Figure 7). The circumference of each participant's forearm was measured to calculate the distance the armband had to be rotated.

This study consisted of three conditions (A, B, C) which are listed in detail in Table 1. In each condition, the Box and Beans test was performed nine times and a questionnaire was conducted. At first condition A was executed, consisting of recording the participant's myoelectric signals in order to train the pattern recognition algorithm. Each of the five movements in both DoFs (resting, hand open, hand close, supination, pronation) and the four combinations (hand open with supination, hand open with pronation, hand close with supination, hand close with pronation) were recorded once for $6.5 \mathrm{~s}$ each. Additionally, the five single movements were recorded two additional times to increase the robustness of the machine learning model. Afterwards, the Box and Beans test was performed to establish a baseline performance.

After finishing condition A, the Myo Armband was

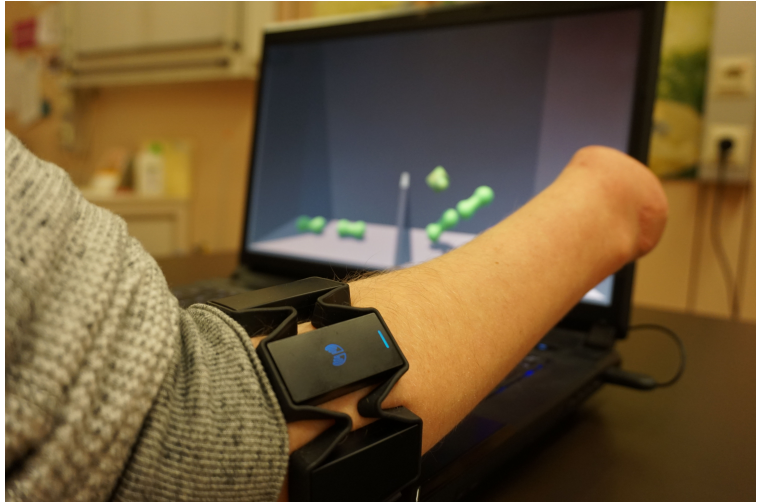

Figure 7: Study set-up: The patient is wearing the Myo armband around his forearm with the elbow resting on the table and facing the computer screen.

rotated by $\mathrm{c}=0.8$ electrodes. In practice, this resulted in shifts around $2 \mathrm{~cm}$, being an upper bound to electrode shifts with custom made sockets reported in the literature (Sebastian Amsüss 2015) (section 2 therein). We investigate an upper bound to ensure that our approach can handle disturbances that severely affect functionality.

Three additional movements - resting, to estimate the shift from baseline activity, as well as hand close and supination - were recorded in this state for $6.5 \mathrm{~s}$ each and the transfer learning algorithm was applied. Note that the data of these three movements would be insufficient to train a new model because several classes are missing (hand open and pronation). Two experimental conditions followed in randomized order:

B) Performing the Box and Beans test nine times using the original model.

C) Performing the Box and Beans test nine times using the result of the transfer learning algorithm together with the original model.

The current condition was concealed to participants by performing an apparently equivalent setup for both conditions. In condition $\mathrm{C}$, the learned transformation was applied to the incoming data, while in condition $\mathrm{B}$, a mock transformation was used that did not change the data.

This study was approved by the ethics committee at the Medical University of Vienna, Austria, under [1301/2015] and all participants provided their consent prior to the study. 


\section{Results}

\subsection{Questionnaire}

The mean scores across all 13 participants of the five subscales of the IMI for all three conditions are displayed in Figure 8. Error bars indicate standard deviation. There was no significant difference between ratings by patients and able-bodied participants. Across all 13 participants, a Kruskal-Wallis test with significance threshold $\alpha=.05$ revealed that participants enjoyed using the control algorithm significantly more during condition A and C $(p=.001)$. Moving the controller in condition $\mathrm{B}$ also required significantly $(p=.01)$ more effort than during condition A and C. Subsequently, this algorithm was rated as significantly less useful for controlling a myoelectric arm prosthesis in condition B compared to condition A and $\mathrm{C}(p=.004)$.

\subsection{Transfer Learning}

The median scores over 9 runs in the Box and Beans test for all participants and all conditions are displayed in Figure 9. The first four bars correspond to amputees, the latter nine to able-bodied participants. Bar height corresponds to median score in conditions A (gray), B (blue, dotted), and C (orange, striped) respectively. Error bars indicate the inter-quartile range.

We compared median scores for conditions B and $\mathrm{C}$ across the nine able-bodied participants using a one-sided Wilcoxon sign-rank test, revealing a significant difference $(p<0.05$ after Bonferroni correction). Additionally, for each of the 13 participants, we compared the 9 Box and Beans test scores in condition $\mathrm{B}$ with the scores in condition $\mathrm{C}$ using a onesided Wilcoxon rank-sum test, revealing significant differences for seven of the 13 participants $(p<0.05$ after Bonferroni correction; also refer to Figure 9). Note that we do not make claims regarding the difference to condition A because this condition was always performed before the other two, such that training effects may confound the statistical analysis.

\section{Discussion and Conclusion}

In this paper, we proposed a novel transfer learning scheme to counteract electrode shifts, introduced the
Table 1: Overview of study procedures

\begin{tabular}{|c||l|c|c|}
\hline Cond. & Data / prosthesis control & Box'n'Beans & Questionnaire \\
\hline A & recording initial movements & 9 times & IMI \\
\hline \multicolumn{1}{|c||}{} & rotate Myo armband, record 3 movements \\
\hline B & use original model & 9 times & IMI \\
\hline C & apply transfer learning & 9 times & IMI \\
\hline
\end{tabular}

virtual Box and Beans test as a novel test of prosthetic proficiency, and we provided the first experimental study to evaluate transfer learning to counteract electrode shifts. Given that the muscle configuration and muscle mass of upper limb amputees differs from able-bodied participants, it is important to evaluate any algorithm which is aimed at clinical application on actual patients. In our study, we evaluated transfer learning both on able-bodied participants and on transradial amputees, and found consistent results for both groups. In particular, we could show significant improvements in Box and Beans score for five out of nine able-bodied participants and two out of four transradial amputees compared to the control condition, indicating that a wide range of transradial prosthesis users could benefit from transfer learning. In no cases did the performance decrease. Further, we observed a significant overall improvement across the able-bodied population. Consistent with score improvements, participants regarded the system after transfer learning as more enjoyable, more effortless in handling and more useful for a potential myoelectric prosthesis control compared to the control condition

The approach as presented in this paper is limited to electrode shifts, specifically transversal shifts in regular electrode grids around the forearm. In case of irregular electrode placements, different transfer functions need to be applied, adjusted to the specific configuration of the electrodes. We also assumed that features change linearly between neighboring electrodes, which is only justified if electrodes are sufficiently dense to make a linear approximation plausible. Therefore, configurations with fewer than eight electrodes may not benefit as much from the presented transfer learning scheme. Also, our approach is limited to cases where electrode shifts act on all electrodes equally, which may not hold in case of very soft materials or disturbances beyond electrode shifts, such as sweat, fatigue, or electrode liftoff. Generalizations to these kinds of disturbances could be possible if a model for the impact exists and can be parametrized in a low-dimensional space. Fi- 
questionnaire results

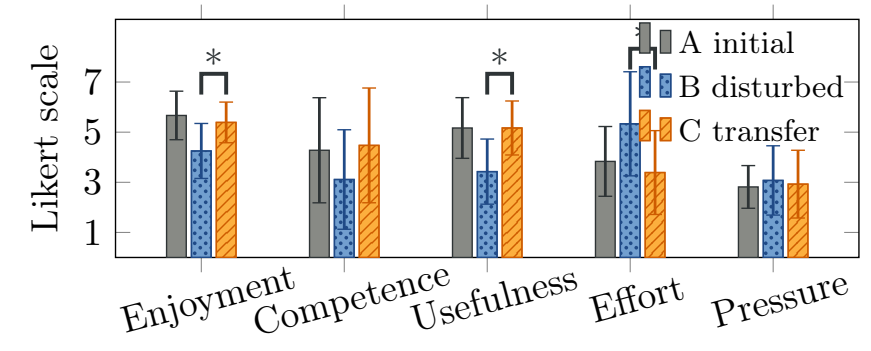

Figure 8: The average response for the questionnaire on a 7-point Likert scale after using the original pattern recognition system (condition A, grey bar), after using the system under electrode shift (condition B, blue dotted bar), after using the system after transfer learning (condition C, orange striped bar). Error bars indicate standard deviation.

nally, the virtual Box and Beans test has its own limitations in neglecting the weight of the prosthesis and the blocks. It is a general limitation for virtual environments that there is neither haptic nor weight related feedback. However, it does not compromise the transfer learning results. Also, we coerced the participants to actively employ a DoF (rotation) that they do not necessarily need during the analogue Box and Block test. We showed that for a low-cost consumer-grade sensor with a moderate number of electrodes, our transfer learning approach offers a data-parsimoneous, fast, easy to implement, and effective way to counteract electrode shifts. This gives reason to hope that transfer learning could improve prosthetic applications in the future, enhancing usability and robustness, and, in turn, patients' lives.

\section{Acknowledgment}

Funding by the DFG under grant number HA2719/62, the CITEC center of excellence (EXC 277), as well as the Christian Doppler Research Foundation of the Austrian Federal Ministry of Science, Research and Economy is gratefully acknowledged.

\section{Literature}

Amsüss, Sebastian (2015). "Robust electromyography based control of multifunctional prostheses of the upper extremity". Dissertation. Georg-August-
Universität Göttingen. URL: https://ediss .unigoettingen.de/handle/11858/00-1735-00000022-5F96-6.

Amsüss, S. et al. (2014). "Self-Correcting Pattern Recognition System of Surface EMG Signals for Upper Limb Prosthesis Control". In: IEEE Transactions on Biomedical Engineering 61.4, pp. 11671176. DOI: 10.1109/TBME. 2013.2296274.

Belter, Joseph T. et al. (2013). "Mechanical design and performance specifications of anthropomorphic prosthetic hands: A review". In: Journal of Rehabilitation Research \& Development 50.5, pp. 599-618. DOI: 10.1682/JRRD.2011.10.0188.

Biddiss, Elaine A. and Tom T. Chau (2007). "Upper limb prosthesis use and abandonment: A survey of the last 25 years". In: Prosthetics and Orthotics International 31.3, pp. 236-257. DOI: $10.1080 /$ 03093640600994581 .

Controzzi, Marco et al. (2017). "The SSSA-MyHand: A Dexterous Lightweight Myoelectric Hand Prosthesis". In: IEEE Transactions on Neural Systems and Rehabilitation Engineering 25.5, pp. 459-468. DOI: 10.1109/TNSRE.2016.2578980.

Daley, Heather et al. (2012). "High density electromyography data of normally limbed and transradial amputee subjects for multifunction prosthetic control". In: Journal of Electromyography and Kinesiology 22.3, pp. 478-484. ISSN: 10506411. DOI: $10.1016 / \mathrm{j} \cdot \mathrm{jelekin} .2011 .12 .012$.

Farina, D. et al. (2014). "The Extraction of Neural Information from the Surface EMG for the Control of Upper-Limb Prostheses: Emerging Avenues and Challenges". In: IEEE Transactions on Neural Systems and Rehabilitation Engineering 22.4, pp. 797-809. DOI: 10.1109/TNSRE. 2014.2305111. Fletcher, R. (1987). Practical Methods of Optimization; (2Nd Ed.) New York, NY, USA: WileyInterscience. ISBN: 0-471-91547-5.

Hahne, J. M. et al. (2015). "Concurrent Adaptation of Human and Machine Improves Simultaneous and Proportional Myoelectric Control". In: IEEE Transactions on Neural Systems and Rehabilitation Engineering 23.4, pp. 618-627. DOI: 10.1109/ TNSRE. 2015.2401134.

Hahne, Janne M., Felix Biebmann, et al. (2014). "Linear and nonlinear regression techniques for simultaneous and proportional myoelectric control". In: IEEE Transactions on Neural Systems and Re- 
amputees

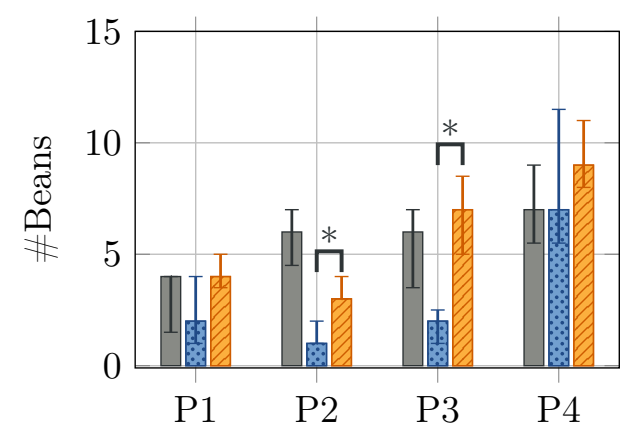

able-bodied participants

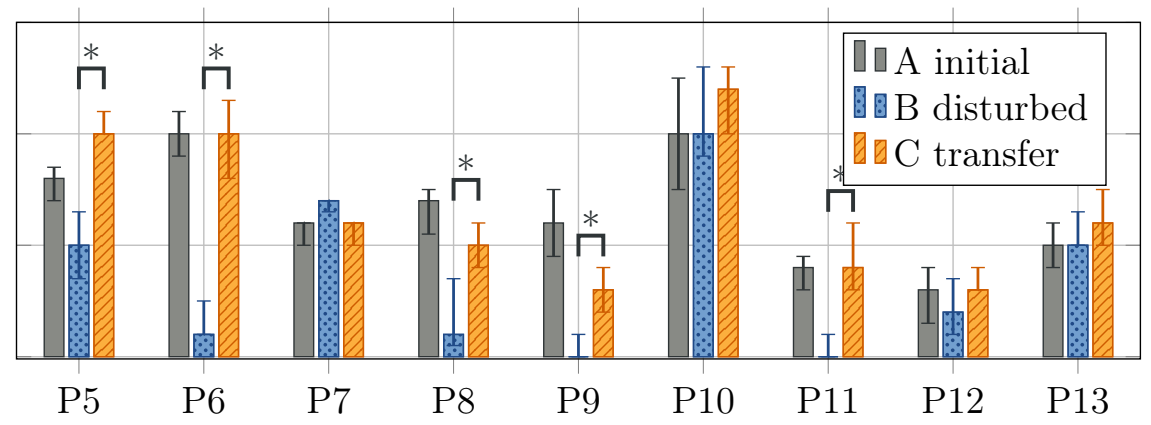

Figure 9: The median number of transferred beans in 60s across nine trials. The result for each patient and participant are shown via three bars, one for the performance using the original pattern recognition system (condition A, gray), one for the performance after electrode shift (condition B, blue, dotted), and one for the performance after transfer learning (condition C, orange, striped). Error bars indicate the lower and upper quartile. Significant differences between conditions B and C are indicated with brackets.

habilitation Engineering 22.2, pp. 269-279. DOI: 10.1109/TNSRE. 2014.2305520.

Hahne, Janne M., Dario Farina, et al. (2016). "A Novel Percutaneous Electrode Implant for Improving Robustness in Advanced Myoelectric Control". In: Frontiers in Neuroscience 10.114. DOI: 10 . 3389/fnins. 2016.00114.

Hargrove, Levi, Kevin Englehart, and Bernard Hudgins (2008). "A training strategy to reduce classification degradation due to electrode displacements in pattern recognition based myoelectric control". In: Biomedical signal processing and control 3.2, pp. 175-180. DOI: 10.1016/j.bspc.2007.11.005.

Hwang, Han-Jeong, Janne Mathias Hahne, and Klaus-Robert Müller (2017). "Real-time robustness evaluation of regression based myoelectric control against arm position change and donning/doffing". In: PLOS ONE 12.11, pp. 1-22. DOI: $10.1371 /$ journal pone.0186318.

Jiang, Ning et al. (2014). "Intuitive, online, simultaneous, and proportional myoelectric control over two degrees-of-freedom in upper limb amputees". In: IEEE Transactions on Neural Systems and Rehabilitation Engineering 22.3, pp. 501-510. DOI: 10.1109/TNSRE. 2013.2278411.

Khushaba, Rami N. et al. (2014). "Towards limb position invariant myoelectric pattern recognition using time-dependent spectral features". In: Neural Networks 55, pp. 42-58. DOI: $10.1016 /$ j.neunet. 2014.03.010.

Mathiowetz, V. et al. (1985). "Adult norms for the Box and Block Test of manual dexterity". In:
American Journal of Occupational Therapy 39(6), pp. 386-391. DOI: 10.5014/ajot.39.6.386.

Muceli, S., N. Jiang, and D. Farina (2014). "Extracting Signals Robust to Electrode Number and Shift for Online Simultaneous and Proportional Myoelectric Control by Factorization Algorithms". In: IEEE Transactions on Neural Systems and Rehabilitation Engineering 22.3, pp. 623-633. DOI: 10 . 1109/TNSRE. 2013.2282898.

Muceli, S., I. Vujaklija, et al. (2017). "A BiologicallyInspired Robust Control System for Myoelectric Control". In: Proceedings of the 3rd International Conference on NeuroRehabilitation (ICNR 2016). Springer, pp. 975-979. DOI: 10.1007/978-3-31946669-9_158.

Ortiz-Catalan, Max et al. (2012). "On the viability of implantable electrodes for the natural control of artificial limbs: Review and discussion". In: Biomedical engineering online 11.1, p. 33. DOI: 10 . 1186/1475-925X-11-33.

Paaßen, Benjamin, Alexander Schulz, Janne Hahne, et al. (2018). "Expectation maximization transfer learning and its application for bionic hand prostheses". In: Neurocomputing 298, pp. 122-133. DOI: 10.1016/j.neucom.2017.11.072.

Paaßen, Benjamin, Alexander Schulz, and Barbara Hammer (2016). "Linear Supervised Transfer Learning for Generalized Matrix LVQ". In: Proceedings of the Workshop New Challenges in Neural Computation (NC ${ }^{2}$ 2016. 4, pp. 11-18.

Pan, Lizhi et al. (2015). "Improving robustness against electrode shift of high density EMG for 
myoelectric control through common spatial patterns". In: Journal of NeuroEngineering and Rehabilitation 12.1, pp. 1-16. DOI: $10.1186 /$ s12984015-0102-9.

Pan, S. J. and Q. Yang (2010). "A Survey on Transfer Learning". In: IEEE Transactions on Knowledge and Data Engineering 22.10, pp. 1345-1359. DOI: 10.1109/TKDE. 2009.191.

Pasquina, Paul F. et al. (2015). "First-in-man demonstration of a fully implanted myoelectric sensors system to control an advanced electromechanical prosthetic hand". In: Journal of Neuroscience Methods 244, pp. 85-93. DOI: $10.1016 / \mathrm{j}$. jneumeth.2014.07.016.

Phinyomark, Angkoon, Rami N. Khushaba, and Erik Scheme (2018). "Feature Extraction and Selection for Myoelectric Control Based on Wearable EMG Sensors". In: Sensors 18.5, p. 1615. DOI: 10.3390/ s18051615.

Prahm, Cosima, Benjamin Paaßen, et al. (2016). "Transfer Learning for Rapid Re-calibration of a Myoelectric Prosthesis After Electrode Shift". In: Proceedings of the 3rd International Conference on NeuroRehabilitation (ICNR 2016). Springer, pp. 153-157. DOI: $10.1007 / 978-3-319-46669-$ 9_28.

Prahm, Cosima, Alexander Schulz, Benjamin Paaßen, et al. (2017). "Echo State Networks as Novel Approach for Low-Cost Myoelectric Control". In: Proceedings of the 16th Conference on Artificial Intelligence in Medicine (AIME 2017). Vol. 10259. Springer. DOI: $10.1007 / 978-$ 3-319-59758-4_40.

Prahm, Cosima, Alexander Schulz, Johannes Schoisswohl, et al. (2019). "Counteracting Electrode Shifts in Upper-Limb Prosthesis Control via Transfer Learning". In: IEEE Transactions on Neural Systems and Rehabilitation Engineering. epub ahead of print. DOI: 10.1109/TNSRE. 2019.2907200.

Prahm, Cosima, Ivan Vujaklija, et al. (2017). "Gamebased rehabilitation for myoelectric prosthesis control". In: JMIR serious games 5.1. DOI: 10.2196/ games.6026.

Roche, Aidan Dominic et al. (2015). "A Structured Rehabilitation Protocol for Improved Multifunctional Prosthetic Control: A Case Study." In: Journal of visualized experiments (105), e52968. DOI: $10.3791 / 52968$
Roche, Aidan D. et al. (2014). "Prosthetic Myoelectric Control Strategies: A Clinical Perspective". In: Current Surgery Reports 2.3, p. 44. DOI: $10.1007 /$ s40137-013-0044-8.

Ryan, Richard M. (1982). "Control and information in the intrapersonal sphere: An extension of cognitive evaluation theory". In: Journal of Personality and Social Psychology 43.3, pp. 450-461. DOI: 10.1037/0022-3514.43.3.450.

Schneider, Petra, Michael Biehl, and Barbara Hammer (2009). "Adaptive Relevance Matrices in Learning Vector Quantization". In: Neural Computation 21.12, pp. 3532-3561. DOI: 10.1162/neco. 2009.11-08-908.

Todd, Kuiken et al. (2009). "Targeted muscle reinnervation for real-time myoelectric control of multifunction artificial arms". In: JAMA 301.6, pp. 619-628. DOI: 10.1001/jama.2009.116.

Vidovic, M. et al. (2015). "Improving the Robustness of Myoelectric Pattern Recognition for Upper Limb Prostheses by Covariate Shift Adaptation". In: IEEE Transactions on Neural Systems and Rehabilitation Engineering 99. DOI: 10.1109/TNSRE. 2015.2492619.

Young, A. J., L. J. Hargrove, and T. A. Kuiken (2011). "The Effects of Electrode Size and Orientation on the Sensitivity of Myoelectric Pattern Recognition Systems to Electrode Shift". In: IEEE Transactions on Biomedical Engineering 58.9, pp. 2537-2544. DOI: 10.1109/TBME . 2011. 2159216.

- (2012). "Improving Myoelectric Pattern Recognition Robustness to Electrode Shift by Changing Interelectrode Distance and Electrode Configuration". In: IEEE Transactions on Biomedical Engineering 59.3, pp. 645-652. DOI: 10.1109/TBME. 2011.2177662. 